\title{
Integrative genomic analyses of ZEB2: Transcriptional regulation of ZEB2 based on SMADs, ETS1, HIF1a, POU/OCT, and NF-kB
}

\author{
MASUKO KATOH ${ }^{1}$ and MASARU KATOH ${ }^{2}$ \\ ${ }^{1}$ M\&M Medical BioInformatics, Hongo 113-0033; ${ }^{2}$ Genetics and Cell Biology Section, \\ National Cancer Center Research Institute, Tokyo 104-0045, Japan \\ Received March 17, 2009; Accepted April 14, 2009
}

DOI: 10.3892/ijo_00000304

\begin{abstract}
Epithelial-to-mesenchymal transition (EMT) is defined as phenotypic change of epithelial cells into mesenchymal cells. EMT, allowing cellular dissociation from epithelial tissues, plays a key role in invasion and metastasis during carcinogenesis as well as in gastrulation and neurulation during embryogenesis. SNAI1/Snail, SNAI2/Slug, ZEB1/8EF1/ ZFHX1A, ZEB2/SIP1/ZFHX1B, TWIST1/TWIST, and TWIST2/DERMO1 are representative EMT regulators. ZEB2 represses transcription of $C D H 1, C L D N 4, C C N D 1, T E R T$, SFRP1, ALPL and miR-200b-200a-429 primary miRNA, and upregulates transcription of mesenchymal markers. ZEB2 is relatively highly expressed in brain corpus callosum and monocytes. ZEB2 is expressed in various types of human tumors, such as breast cancer, gastric cancer, and pancreatic cancer. TGFß, TNF $\alpha$, IL1, AKT and hypoxia signals are involved in ZEB2 upregulation and EMT induction; however precise mechanisms of $Z E B 2$ transcription remained unclear. Here, refined integrative genomic analyses of $Z E B 2$ gene were carried out. $Z E B 2$ was co-expressed with POU3F2 (BRN2) and POU $3 F 3$ (BRN1) in brain corpus callosum, spinal cord, and fetal brain, whereas ZEB2 was co-expressed with $P O U 2 F 2$ (OCT2) in monocytes. Ets-Smad-binding CGGAGAC motif, bHLH-binding site, and POU/OCT-binding site within proximal promoter region, and NF- $\mathrm{B}$-binding site within intron 2 were completely conserved in human $Z E B 2$, chimpanzee ZEB2, cow ZEB2, mouse Zeb2, rat Zeb2, and chicken zeb2 genes. In addition, HIF1 $\alpha$-binding site within proximal promoter region was conserved in mammalian $Z E B 2$ orthologs. Consensus binding site for Hedgehog effector GLI was not identified within or adjacent to the 7-kb regions of human $Z E B 2$ gene. TGFß, TNF $\alpha$, IL1, and hypoxia signals directly upregulate $Z E B 2$ to induce EMT, growth arrest, and
\end{abstract}

Correspondence to: Dr Masaru Katoh, Genetics and Cell Biology Section, National Cancer Center, 5-1-1 Tsukiji, Chuo Ward, Tokyo 104-0045, Japan

E-mail: mkatoh-kkr@umin.ac.jp

Key words: epithelial-to-mesenchymal transition, cellular polarity, telomerase, breast cancer, gastric cancer, pancreatic cancer, peritoneal dissemination, bioinformatics, systems biology, personalized medicine senescence, whereas Hedgehog signals indirectly upregulate ZEB2 via TGFß. Together these facts indicate that ZEB2, occupying the crossroads of inflammation, aging and carcinogenesis, is an important target for drug discovery.

\section{Introduction}

Epithelial-to-mesenchymal transition (EMT) is defined as phenotypic change of epithelial cells into mesenchymal cells (1-4). Epithelial cells with E-cadherin expression are tightly held together with uniform neighboring cells to move as a sheet en block, whereas mesenchymal cells without E-cadherin expression are loosely connected with diverse neighboring cells to move individually. EMT, allowing cellular dissociation from epithelial tissues, plays a key role in invasion and metastasis during carcinogenesis as well as in gastrulation and neurulation during embryogenesis.

Downregulation of E-cadherin at the adherens junction and Claudin at the tight junction leads to the loss of epithelial phenotype, while upregulation of $\mathrm{N}$-cadherin and Vimentin leads to the acquisition of mesenchymal phenotype. SNAI1/ Snail (5), SNAI2/Slug (6), SNAI3 (7), ZEB1/8EF1/ZFHX1A (8), ZEB2/SIP1/ZFHX1B (9), TWIST1/TWIST (10), and TWIST2/DERMO1 (11) are representative EMT regulators. Upregulation of EMT regulators in tumor cells is associated with more malignant phenotypes in a variety of human cancer (12-16).

ZEB2 and ZEB1 are transcription factors with common domain architecture consisting of the p300-binding domain and four zinc finger domains in the N-terminal region, Smadbinding domain, Homeo domain and CtBP-binding domain in the middle region, and three zinc finger domains in the C-terminal region $(17,18)$. ZEB2 is involved in transcriptional repression of CDH1 (9), ALPL (19), TERT (20), CLDN4 (21), CCND1 (22), SFRP1 (23) and miR-200b-200a-429 primary miRNA (24) through the recruitment of CtBP co-repressor. ZEB2 is also involved in transcriptional activation of mesenchymal markers through the recruitment of p300 coactivator in cooperation with SMAD complex or through indirect mechanisms.

ZEB2 is expressed in various types of human tumors, including breast cancer $(9,25-27)$, gastric cancer $(13,15)$, colorectal cancer (28), liver cancer $(29,30)$, ovarian cancer (25), oral squamous cell carcinoma $(31,32)$, pancreatic cancer (33), and kidney cancer (34), as summarized in Table I. 
Table I. ZEB2 expression in human tumors.

\begin{tabular}{lll}
\hline Human cancer & Expression and mechanism & Author/(Refs.) \\
\hline Breast cancer & Upregulation in cell lines & Comijn et al (9) \\
& Upregulation in primary tumors & Elloul et al (25) \\
& Upregulation in primary tumors & Gregory et al (26) \\
& Upregulation due to promoter hypomethylation & Rodenhiser et al (27) \\
Gastric cancer & Upregulation in primary tumors & Rosivatz et al (13) \\
Colorectal cancer & Upregulation in primary tumors & Castro Alves et al (15) \\
Liver cancer & Upregulation in cell lines & Guaita et al (28) \\
Ovarian cancer & Upregulation in cell lines & Miyoshi et al (29) \\
Oral SCC & Upregulation in primary tumors & Elloul et al (25) \\
Pancreatic cancer & Upregulation in primary tumors & Maeda et al (31) \\
Kidney cancer & Upregulation in primary tumors & Taki et al (32)
\end{tabular}

Because ZEB2 is one of key molecules involved in carcinogenesis, refined integrative genomic analyses of ZEB2 gene were carried out in this study to elucidate the mechanisms of ZEB2 transcription.

\section{Materials and methods}

Comparative genomic analyses. Human genome sequences corresponding to human ZEB2 RefSeq (NM_014795.2) were searched for by using BLAST programs, as previously described $(35,36)$. ZEB2 expressed sequence tags (ESTs) were also searched for to identify $Z E B 2$ splicing variants $(37,38)$. Conserved transcription factor-binding sites within $Z E B 2$ promoters were then searched for based on manual inspection, as previously described $(39,40)$.

Regulatory network analyses. Literature on ZEB2, NF-кB, TGFß, Notch and Hedgehog signaling molecules in PubMed and Medline databases was critically evaluated to extract knowledge on the regulation of NF-кB, SMAD, CSL, GLI and FOX transcription factors. The mechanisms of ZEB2 transcription were then investigated based on our data of conserved transcription factor-binding sites within ZEB2 promoters and in-house knowledgebase of transcription factors regulated by the stem-cell signaling network.

\section{Results}

ZEB2 splicing variants transcribed by using alternative promoters. BLAST programs using ZEB2 RefSeq (NM_014795.2) as a query sequence revealed that the $Z E B 2$ gene was located within human genome sequences AC009951.10 and AC010130.12. Human ZEB2 gene, consisting of 10 exons, was found to be about $132 \mathrm{~kb}$ in size, and intron 2 was about $87 \mathrm{~kb}$ in size (Fig. 1). BLAST programs using human ZEB2 genome sequence as a query sequence revealed that more than 50 ESTs were transcribed based on the major promoter, 5 '-adjacent to exon 1, and that two ESTs were transcribed based on the minor promoter, 5'-adjacent to exon 2 (data not shown).

Comparative genomic analyses of ZEB2 orthologs. BLAST programs using human $Z E B 2$ genome sequence as a query sequence next revealed that chimpanzee $Z E B 2$ gene, cow $Z E B 2$ gene, mouse Zeb2 gene, rat Zeb2 gene, and chicken zeb2 gene were located within NW_001232106.1, NW_001494615.2, NT_039206.7, NW_047654.1, and NW_001471733.1 genome sequences, respectively. ZEB2 orthologs were well conserved not only within exonic regions, but also within the major promoter region and intronic regions (data not shown).

TGFß signaling and ZEB2. TGFß-binding to TGFß receptors leads to autophosphorylation of cytoplasmic serine-kinase domain of TGFß receptors, which results in phosphorylation of Smad2 or Smad3 signal transducer, and transcriptional activation of TGFß-target genes based on the Smad2-Smad4 or Smad3-Smad4 complex $(41,42)$. TGFß signaling cascades are involved in ZEB2 upregulation and EMT $(20,43,44)$; however, precise mechanisms of TGFß-induced $Z E B 2$ upregulation remain to be elucidated.

Multiple Smad-binding elements were identified within human ZEB2 promoter based on manual inspection (data not shown). TGFß signals and Ets1 are known to synergistically upregulate transcription of target genes involved in invasion and metastasis (45). The CGGAGAC motif, corresponding to 
$2 q 22.3$
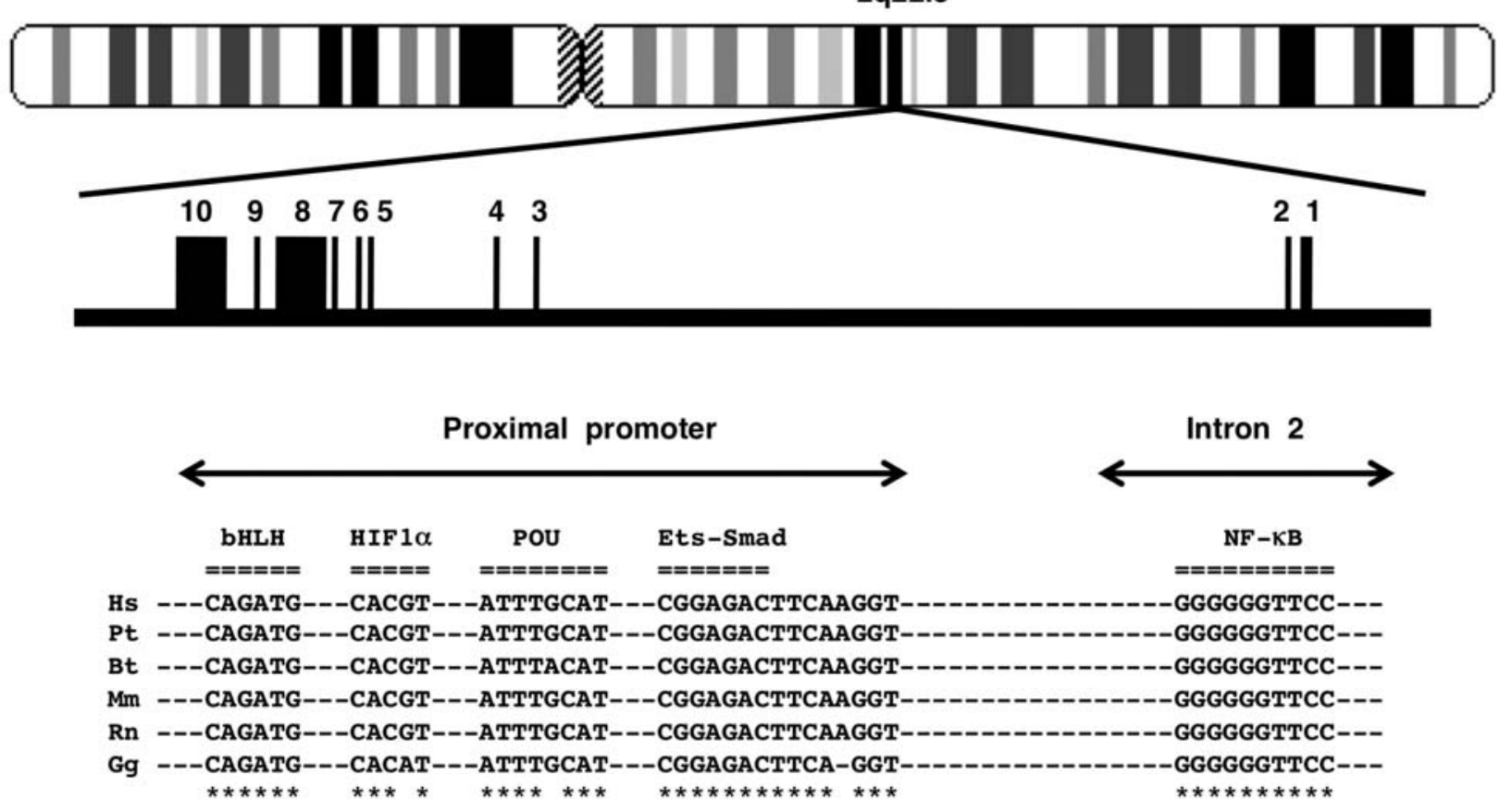

Figure 1. Integrative genomic analyses of $Z E B 2$. Schematic representation of the ZEB2 gene at human chromosome $2 \mathrm{q} 22.3$ is shown in the upper part. $Z E B 2$ gene consists of 10 exons, and intron 2 is about $87 \mathrm{~kb}$ in size. Conserved transcription factor-binding sites within $Z E B 2$ regulatory regions are shown in the lower part. Hs, human; Pt, chimpanzee; Bt, cow; Mm, mouse; Rn, rat; Gg, chicken.

Ets- and Smad-binding sites located at the -8 bp position from the transcriptional start site (TSS) of human ZEB2 RefSeq was completely conserved in chimpanzee $Z E B 2$, cow $Z E B 2$, mouse Zeb2, rat Zeb2, and chicken zeb2 genes (Fig. 1).

Hedgehog signaling and ZEB2. Hedgehog-binding to Patched receptors leads to activation of Smoothened signaling, which results in transcriptional activation of Hedgehog target genes via GLI activators (46-48). Hedgehog signaling cascades are also involved in EMT $(4,49)$. Consensus GLI-binding site was not identified within the $10-\mathrm{kb}$ upstream region from the TSS of human ZEB2 RefSeq as well as within the ZEB2 gene (data not shown). Therefore, Hedgehog-GLI signaling cascades are predicted to be involved in indirect rather than direct transcriptional activation of $Z E B 2$.

Hypoxia signaling and ZEB2. HIF1 complex, consisting of HIF1 $\alpha$ and HIF1ß/ARNT, is involved in cellular response to hypoxia through transcriptional regulation of genes involved in energy metabolism, angiogenesis, apoptosis, and EMT $(50,51)$. HIF1 $\alpha$ undergoes ubiquitin-mediated proteasomal degradation under normoxic condition to inhibit HIF1 activity, whereas HIF1 $\alpha$ is stabilized under hypoxic condition to activate HIF1 activity. Hypoxia-induced HIF1 $\alpha$ upregulation leads to invasion and metastasis of tumor cells through upregulation of EMT regulators, such as SNAI1, ZEB1, ZEB2, and TWIST1 $(52,53)$.

Because the transcriptional mechanisms of HIF1-induced ZEB2 upregulation remain unknown, HIF1 $\alpha$-binding site (hypoxia response element) within $Z E B 2$ promoter was next searched for. HIF1 $\alpha$-binding site was identified at the -876-bp position from the TSS of human ZEB2 RefSeq. The HIF1 $\alpha-$ binding site within the proximal promoter region of human
ZEB2 gene was conserved in chimpanzee ZEB2, cow ZEB2, mouse Zeb2, and rat Zeb2 genes (Fig. 1).

$N F-\kappa B$ signaling and $Z E B 2 . N F-\kappa B$ is a key transcription factor involved in inflammation and carcinogenesis (54-56). Because IкB tethers $\mathrm{NF}-\kappa \mathrm{B}$ in the cytoplasm to prevent its nuclear translocation and subsequent transcriptional activation of target genes, IKK-induced phosphorylation of IкB leads to its ubiquitin-mediated proteasomal degradation, and NF-кB activation. TNF $\alpha$, IL1, and TGFß signals activate IKK via MAP3K7, whereas PI3K signals activate IKK via AKT.

Chua et al reported that TNF $\alpha$, IL1 and AKT induce NF- $\mathrm{BB}$ activation, ZEB2 upregulation and EMT in human MCF-10A cells, while TNF $\alpha$ and IL1 induce ZEB2 upregulation in human MD-231 cells (57). Julien et al reported that NF-кB-binding site is not located within the human ZEB2 promoter (58). We confirmed the absence of NF- $\mathrm{KB}$-binding site within the $10-\mathrm{kb}$ upstream region from the TSS of human ZEB2 RefSeq (data not shown). Moreover, we identified an NF-кB-binding site within the proximal promoter region of the mouse Zeb2 gene; however, the NF-кB-binding site within the mouse Zeb2 promoter was not conserved in human ZEB2 and rat Zeb2 promoters (data not shown).

To elucidate the mechanisms of NF- $\mathrm{BB}$-mediated human $Z E B 2$ upregulation, we next searched for NF- $\mathrm{KB}$-binding sites within the $Z E B 2$ gene or the 3 -adjacent region of the $Z E B 2$ gene. Triple NF-кB-binding sites were identified within intron 2 of the human ZEB2 gene (data not shown). The first NF-кBbinding site at the position about $59-\mathrm{kb}$ downstream from the TSS of human ZEB2 RefSeq was conserved in chimpanzee $Z E B 2$, cow ZEB2, mouse Zeb2, rat Zeb2, and chicken zeb2 genes (Fig. 1). The second NF-кB-binding site at the position about 66-kb down stream from the TSS of human ZEB2 


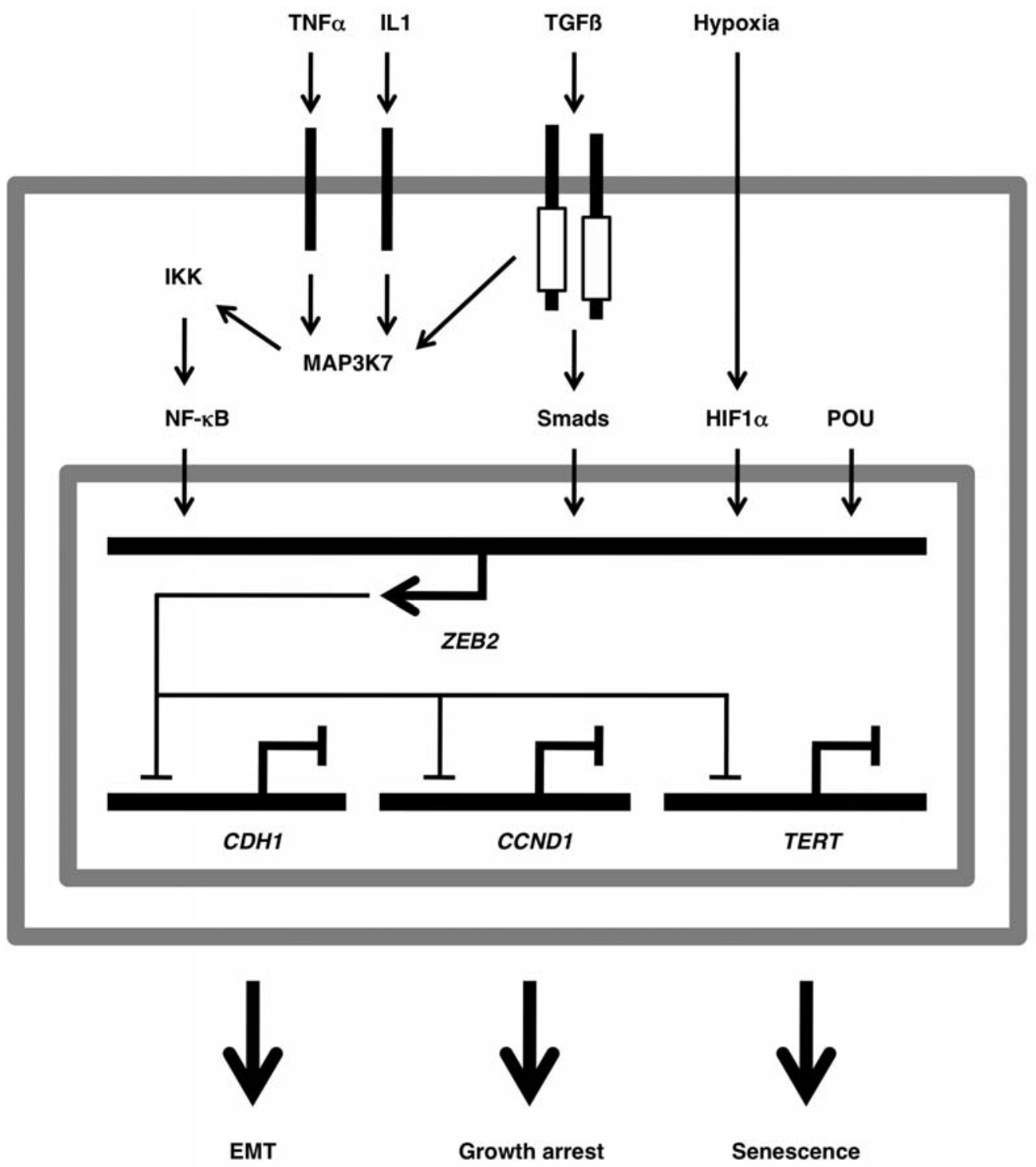

Figure 2. ZEB2 at the crossroads of inflammation, aging and carcinogenesis. Transcriptional mechanisms of ZEB2 mRNA and effects of ZEB2 protein are summarized.

RefSeq was conserved in chimpanzee ZEB2, cow ZEB2, and mouse Zeb2 genes, while the third NF-кB-binding site at the position about 72-kb downstream from the TSS of human ZEB2 RefSeq was conserved in chimpanzee ZEB2, and mouse Zeb2 genes (data not shown). Because the first NF-кB-binding site within intron 2 of $Z E B 2$ orthologs was ultra-conserved in mammals and chicken (Fig. 1), it was concluded that the

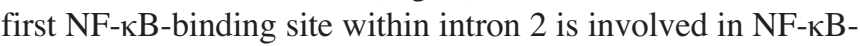
mediated ZEB2 upregulation.

Other transcription factor-binding sites. After the identification of evolutionarily conserved Ets-Smad-, HIF1 $\alpha$ - and NF-кBbinding sites, we then searched for other transcription factorbinding sites by using the Genetyx program. bHLH-binding site at the -940 bp position from the TSS of human ZEB2 RefSeq was conserved in chimpanzee $Z E B 2$, cow $Z E B 2$, mouse Zeb2, rat Zeb2, and chicken zeb2 genes, while POU (OCT)-binding site at the -876 bp position from the TSS of human ZEB2 RefSeq was conserved in chimpanzee ZEB2, cow ZEB2, mouse Zeb2, and rat Zeb2 genes (Fig. 1).

\section{Discussion}

Refined integrative genomic analyses of $Z E B 2$ gene were carried out in this study. Ets-Smad-binding CGGAGAC motif, bHLH- and POU/OCT-binding sites within the proximal promoter region, and NF-кB-binding site within intron 2 were completely conserved in human $Z E B 2$, chimpanzee $Z E B 2$, cow ZEB2, mouse Zeb2, rat Zeb2, and chicken zeb2 genes (Fig. 1). In addition, HIF1 $\alpha$-binding site within the proximal promoter region was conserved in mammalian ZEB2 orthologs (Fig. 1). TGFß signals activate $Z E B 2$ transcription via Smads, Ets1, and NF-кB; TNF $\alpha$ and IL1 signals activate ZEB2 transcription via NF-кB; Hypoxia signals activate ZEB2 transcription via HIF1 $\alpha$ (Fig. 2). Together these facts indicate that TGFß, TNF $\alpha$, IL1, and hypoxia signals directly upregulate ZEB2.

Hedgehog-GLI signaling cascades cross-talk with TGFß, WNT, FGF, and Notch signaling cascades to constitute the stem-cell signaling network (59-62). Consensus GLI-binding site was not identified within or adjacent to the $7-\mathrm{kb}$ regions 
of human ZEB2 gene (data not shown). Because Hedgehog signals promote conversion of latent TGFß to active TGFß via Integrin $\alpha v ß 6$ upregulation (63), Hedgehog signals are able to induce $Z E B 2$ upregulation indirectly through TGFß signaling cascades. TGFß signals directly upregulate $Z E B 2$ (Fig. 2), whereas Hedgehog signals are predicted to indirectly upregulate $Z E B 2$ via TGFß.

POU-binding site within the proximal promoter region of human ZEB2 gene was conserved in mammals and chicken (Fig. 1). To investigate the relationship between POU-domain transcription factor and $Z E B 2$ transcription, POU family members co-expressed with $Z E B 2$ were also searched for in this study. POU3F2 (BRN2) and POU3F3 (BRN1) were coexpressed with $Z E B 2$ in brain corpus callosum, spinal cord, and fetal brain, whereas $P O U 2 F 2$ (OCT2) was co-expressed with ZEB2 in monocytes (RefEX database). Involvement of POU family members in $Z E B 2$ transcription in corpus callosum and monocytes should be further investigated in the future.

Heterozygous deletions or truncating mutations of human ZEB2 gene give rise to Mowat-Wilson syndrome, manifesting mental retardation, epilepsy, and variable congenital malformations including microcephaly, agenesis of the corpus callosum, Hirschsprung disease, congenital heart disease, hypospadias, genitourinary anomalies, short stature, and characteristic facial features (64). Mouse Zeb2 is expressed in neural crest and neural epithelium at embryonic day 8.5, and Zeb2 knockout mice show phenotypes similar to human Mowat-Wilson syndrome (65). Because ZEB2 is one of key regulators of EMT, germline mutations of the ZEB2 gene results in multiple congenital malformations due to the migratory failure of neural crest cells.

Monocytes differentiate into macrophages or dendritic cells to function as antigen presenting cells regulating innate and adaptive immune responses $(66,67)$. ZEB2 is more highly expressed in monocytes than in macrophages, whereas Smad7, functioning as an inhibitor for Smad2 and Smad3, is relatively higher expressed in macrophages than in monocytes (RefEX database). Because cellular adhesion and proliferation are promoted during differentiation of monocytes into macrophages (68), Smad7-mediated inhibition of TGFß signaling leads to ZEB2 downregulation to induce phenotypic changes from monocytes to macrophages.

EMT in physiological conditions is associated with dedifferentiation and growth arrest (69). ZEB2 induces direct transcriptional repression of the CCND1 gene, encoding Cyclin D1 (22), and TERT gene, encoding catalytic subunit of telomerase (20). Because constitutive downregulation of Cyclin D1 and telomerase is not preferable for tumor cells, mesenchymalepithelial transition (MET) accompanied by ZEB2 downregulation should occur in tumor cells at the metastasized organs to recover the expression of Cyclin D1 and telomerase.

ZEB2 also represses the SFRPI gene, encoding an endogenous WNT signaling inhibitor (23). Hedgehog signals induce direct upregulation of SFRP1 gene in the earlier phase (70-72), whereas Hedgehog signals might induce indirect down-regulation of SFRPl gene in the later phase through the TGFß-ZEB2 signaling cascade in some situations.

This study demonstrated that TGFß, TNF $\alpha$, IL1, and hypoxia signals directly upregulate $Z E B 2$ to induce EMT, growth arrest, and senescence. Personalized medicine is gradually gaining practicality in parallel with the development of next-generation sequence technology and the peta-scale super-computer (73). Because ZEB2 occupies the crossroads of inflammation, aging and carcinogenesis, ZEB2 should be an important target for drug discovery to promote personalized medicine.

\section{References}

1. Thiery JP: Epithelial-mesenchymal transitions in tumour progression. Nat Rev Cancer 2: 442-454, 2002.

2. Barrallo-Gimeno A and Nieto MA: The Snail genes as inducers of cell movement and survival: implications in development and cancer. Development 132: 3151-3161, 2005.

3. Bailey J, Singh PK and Hollingsworth MA: Cancer metastasis facilitated by developmental pathways: Sonic hedgehog, Notch, and bone morphogenetic proteins. J Cell Biochem 102: 829-839, 2007.

4. Katoh Y and Katoh M: Hedgehog signaling, epithelial-tomesenchymal transition and miRNA. Int J Mol Med 22: 271-275, 2008.

5. Cano A, Pérez-Moreno MA, Rodrigo I, et al: The transcription factor Snail controls epithelial-mesenchymal transitions by repressing E-cadherin expression. Nat Cell Biol 2: 76-83, 2000.

6. Savagner P, Yamada KM and Thiery JP: The zinc-finger protein Slug causes desmosome dissociation, an initial and necessary step for growth factor-induced epithelial-mesenchymal transition. J Cell Biol 137: 1403-1419, 1997

7. Katoh $M$ and Katoh M: Identification and characterization of human SNAIL3 (SNAI3) gene in silico. Int J Mol Med 11: 383-388, 2003.

8. Eger A, Aigner K, Sonderegger S, et al: $\delta \mathrm{EF} 1$ is a transcriptional repressor of E-cadherin and regulates epithelial plasticity in breast cancer cells. Oncogene 24: 2375-2385, 2005.

9. Comijn J, Berx G, Vermassen P, et al: The two-handed E box binding zinc finger protein SIP1 downregulates E-cadherin and induces invasion. Mol Cell 7: 1267-1278, 2001.

10. Yang J, Mani SA, Donaher JL, et al: Twist, a master regulator of morphogenesis, plays an essential role in tumor metastasis. Cell 117: 927-939, 2004

11. Ansieau S, Bastid J, Doreau A, et al: Induction of EMT by Twist proteins as a collateral effect of tumor-promoting inactivation of premature senescence. Cancer Cell 14: 79-89, 2008.

12. Blanco MJ, Moreno-Bueno G, Sarrio D, et al: Correlation of Snail expression with histological grade and lymph node status in breast carcinomas. Oncogene 21: 3241-3246, 2002.

13. Rosivatz E, Becker I, Specht K, et al: Differential expression of the EMT regulators Snail, SIP1, and Twist in gastric cancer. Am J Pathol 161: 1881-1891, 2002.

14. Katoh M: Epithelial-mesenchymal transition in gastric cancer. Int J Oncol 27: 1677-1683, 2005.

15. Castro Alves C, Rosivatz E, Schott C, et al: Slug is overexpressed in gastric carcinomas and may act synergistically with SIP1 and Snail in the down-regulation of E-cadherin. J Pathol 211: 507-515, 2007.

16. Peinado H, Olmeda D and Cano A: Snail, Zeb and bHLH factors in tumour progression: an alliance against the epithelial phenotype? Nat Rev Cancer 7: 415-428, 2007.

17. Remacle JE, Kraft H, Lerchner W, et al: New mode of DNA binding of multi-zinc finger transcription factors: $\delta E F 1$ family members bind with two hands to two target sites. EMBO J 18: 5073-5084, 1999.

18. Postigo AA, Depp JL, Taylor JJ and Kroll KL: Regulation of Smad signaling through a differential recruitment of coactivators and corepressors by ZEB proteins. EMBO J 22: 2453-2462, 2003.

19. Tylzanowski P, Verschueren K, Huylebroeck D and Luyten FP: SIP1 is a repressor of LBK-ALP transcription in bone morphogenetic protein-induced osteogenic differentiation of $\mathrm{C} 2 \mathrm{C} 12$ cells. J Biol Chem 276: 40001-40007, 2001.

20. Lin SY and Elledge SJ: Multiple tumor suppressor pathways negatively regulate telomerase. Cell 113: 881-889, 2003.

21. Vandewalle C, Comijn J, De Craene B, et al: SIP1/ZEB2 induces EMT by repressing genes of different epithelial cell-cell junctions. Nucleic Acids Res 33: 6566-6578, 2005.

22. Mejlvang J, Kriajevska M, Vandewalle C, et al: Direct repression of Cyclin D1 by SIP1 attenuates cell cycle progression in cells undergoing an epithelial mesenchymal transition. Mol Biol Cell 18: 4615-4624, 2007. 
23. Miquelajauregui A, van de Putte T, Polyakov A, et al: SIP1/ Zfhx $1 \mathrm{~b}$ acts upstream of Wnt signaling in the mouse hippocampus and controls its formation. Proc Natl Acad Sci USA 104: 12919-12924, 2007

24. Bracken CP, Gregory PA, Kolesnikoff N, et al: A doublenegative feedback loop between ZEB1-SIP1 and the micro RNA-200 family regulates epithelial-mesenchymal transition. Cancer Res 68: 7846-7854, 2008.

25. Elloul S, Elstrand MB, Nesland JM, et al: Snail, Slug, and Smad-interacting protein 1 as novel parameters of disease aggressiveness in metastatic ovarian and breast carcinoma. Cancer 103: 1631-1643, 2005.

26. Gregory PA, Bert AG, Paterson EL, et al: The miR-200 family and miR-205 regulate epithelial to mesenchymal transition by targeting ZEB1 and SIP1. Nat Cell Biol 10: 593-601, 2008.

27. Rodenhiser DI, Andrews J, Kennette W, et al: Epigenetic mapping and functional analysis in a breast cancer metastasis model using whole-genome promoter tiling microarrays. Breast Cancer Res 10: R62, 2008.

28. Guaita S, Puig I, Francí C, et al: Snail induction of epithelial to mesenchymal transition in tumor cells is accompanied by $M U C 1$ repression and ZEB1 expression. J Biol Chem 277: 39209-39216, 2002.

29. Miyoshi A, Kitajima Y, Sumi K, et al: Snail and SIP1 increase cancer invasion by upregulating MMP family in hepatocellular carcinoma cells. Br J Cancer 90: 1265-1273, 2004.

30. Ozturk N, Erdal E, Mumcuoglu M, et al: Reprogramming of replicative senescence in hepatocellular carcinoma-derived cells. Proc Natl Acad Sci USA 103: 2178-2183, 2006.

31. Maeda G, Chiba T, Okazaki M, et al: Expression of SIP1 in oral squamous cell carcinomas. Int J Oncol 27: 1535-1541, 2005.

32. Taki M, Verschueren K, Yokoyama K, Nagayama M and Kamata N: Involvement of ETS1 transcription factor in inducing MMP2 expression by EMT in human squamous carcinoma cells. Int J Oncol 28: 487-496, 2006.

33. Imamichi Y, König A, Gress T and Menke A: Collagen type Iinduced Smad-interacting protein 1 expression downregulates E-cadherin in pancreatic cancer. Oncogene 26: 2381-2385, 2007

34. Nakada C, Matsuura K, Tsukamoto Y, et al: Genome-wide microRNA expression profiling in renal cell carcinoma: significant down-regulation of miR-141 and miR-200c. J Pathol 216: 418-427, 2008

35. Katoh Y and Katoh M: Conserved POU-binding site linked to SP1-binding site within FZD5 promoter. Int J Oncol 30: 751-755, 2007.

36. Katoh $\mathrm{M}$ and Katoh $\mathrm{M}$ : Comparative integromics on FZD7 orthologs. Int J Mol Med 19: 529-533, 2007.

37. Katoh $\mathrm{M}$ and Katoh $\mathrm{M}$ : Conserved POU/OCT- and GATAbinding sites in 5'-flanking promoter region of mammalian WNT8B orthologs. Int J Oncol 30: 1273-1277, 2007.

38. Katoh Y and Katoh M: Comparative integromics on JMJD2A, JMJD2B and JMJD2C. Int J Mol Med 20: 269-273, 2007.

39. Katoh $\mathrm{M}$ and Katoh $\mathrm{M}$ : Integrative genomic analyses on HES/ HEY family. Int J Oncol 31: 461-466, 2007

40. Katoh Y and Katoh M: Integrative genomic analyses on GLI2. Int J Oncol 33: 881-886, 2008.

41. Bierie B and Moses HL: Tumour microenvironment: TGFß: the molecular Jekyll and Hyde of cancer. Nat Rev Cancer 6: 506-520, 2006.

42. Massagué J: TGFß in cancer. Cell 134: 215-230, 2008.

43. Zwijsen A, van Grunsven LA, Bosman EA, et al: TGFß signalling in vitro and in vivo: Activin ligand-receptor interaction, Smad5 in vasculogenesis, and repression of target genes by the $\delta E F 1 /$ ZEB-related SIP1 in the vertebrate embryo. Mol Cell Endocrinol 180: 13-24, 2001

44. Postigo AA: Opposing functions of $\mathrm{ZEB}$ proteins in the regulation of the TGFß/BMP signaling pathway. EMBO J 22: 2443-2452, 2003

45. Lindemann RK, Ballschmieter P, Nordheim A and Dittmer J: TGFB regulates PTHrP/PTHLH expression in MDA-MB-231 breast cancer cells through a novel Smad/Ets synergism. J Biol Chem 276: 46661-46670, 2001

46. Beachy PA, Karhadkar SS and Berman DM: Tissue repair and stem cell renewal in carcinogenesis. Nature 432: 324-331, 2004.

47. Hooper JF and Scott MP: Communicating with Hedgehogs. Nat Rev Mol Cell Biol 6: 306-317, 2005
48. Katoh Y and Katoh M: Hedgehog signaling in gastric cancer. Cancer Biol Ther 4: 1050-1054, 2005

49. Li X, Deng W, Nail CD, et al: Snail induction is an early response to Gli1 that determines the efficiency of epithelial transformation. Oncogene 25: 609-621, 2006.

50. Wang G, Jiang B, Rue EA and Semenza GL: HIF1 $\alpha$ is a bHLHPAS heterodimer regulated by cellular $\mathrm{O}_{2}$ tension. Proc Natl Acad Sci USA 92: 5510-5514, 1995.

51. Dewhirst MW, Cao Y and Moeller B: Cycling hypoxia and free radicals regulate angiogenesis and radiotherapy response. Nat Rev Cancer 8: 425-437, 2008.

52. Krishnamachary B, Zagzag D, Nagasawa H, et al: HIF1 dependent repression of E-cadherin in von Hippel-Lindau tumor suppressor-null renal cell carcinoma mediated by TCF3, ZFHX1A, and ZFHX1B. Cancer Res 66: 2725-2731, 2006.

53. Yang MH, Wu MZ, Chiou SH, et al: Direct regulation of TWIST by HIF1 $\alpha$ promotes metastasis. Nat Cell Biol 10: 295-305, 2008.

54. Karin $\mathrm{M}$ : $\mathrm{NF}-\kappa \mathrm{B}$ in cancer development and progression. Nature 441: 431-436, 2006.

55. Katoh M: Dysregulation of stem cell signaling network due to germline mutation, SNP, Helicobacter pylori infection, epigenetic change, and genetic alteration in gastric cancer. Cancer Biol Ther 6: 832-839, 2007

56. Hayden MS and Ghosh S: Shared principles in NF-кB signaling. Cell 132: 344-362, 2008.

57. Chua HL, Bhat-Nakshatri P, Clare SE, Morimiya A, Badve S and Nakshatri $\mathrm{H}$ : $\mathrm{NF}-\kappa \mathrm{B}$ represses E-cadherin expression and enhances epithelial to mesenchymal transition of mammary epithelial cells: potential involvement of ZEB1 and ZEB2. Oncogene 26: 711-724, 2007.

58. Julien S, Puig I, Caretti E, et al: Activation of NF- $\kappa$ B by Akt upregulates Snail expression and induces EMT. Oncogene 26: 7445-7456, 2007.

59. Garciadiego-Cazares D, Rosales C, Katoh M and ChimalMonroy J: Coordination of chondrocyte differentiation and joint formation by $\alpha 5 \beta 1$ integrin in the developing appendicular skeleton. Development 131: 4735-4742, 2004

60. Katoh M: Networking of WNT, FGF, Notch, BMP, and Hedgehog signaling pathways during carcinogenesis. Stem Cell Rev 3: 30-38, 2007.

61. Katoh M and Katoh M: WNT signaling pathway and stem cell signaling network. Clin Cancer Res 13: 4042-4045, 2007.

62. Katoh M: WNT signaling in stem cell biology and regenerative medicine. Curr Drug Targets 9: 565-570, 2008.

63. Marsh D, Dickinson S, Neill GW, Marshall JF, Hart IR and Thomas GJ: $\alpha \mathrm{v} ß 6$ Integrin promotes the invasion of morphoeic basal cell carcinoma through stromal modulation. Cancer Res 68: 3295-3303, 2008

64. Mowat DR, Wilson MJ and Goossens M: Mowat-Wilson syndrome. J Med Genet 40: 305-310, 2003.

65. Van de Putte T, Maruhashi M, Francis A, et al: Mice lacking Zeb2 gene reveal a role for multiple neural crest cell defects in the etiology of Hirschsprung disease-mental retardation syndrome. Am J Hum Genet 72: 465-470, 2003.

66. Lehtonen A, Ahlfors H, Veckman V, Miettinen M, Lahesmaa R and Julkunen I: Gene expression profiling during differentiation of human monocytes to macrophages or dendritic cells. J Leukoc Biol 82: 710-720, 2007.

67. Conti L and Gessani S: GM-CSF in the generation of dendritic cells from human blood monocyte precursors: recent advances. Immunobiology 213: 859-870, 2008.

68. Hamilton JA: GM-CSF in inflammation and autoimmunity. Trends Immunol 23: 403-408, 2002.

69. Burstyn-Cohen $\mathrm{T}$ and Kalcheim $\mathrm{C}$ : Association between cell cycle and neural crest delamination through specific regulation of G1/S transition. Dev Cell 3: 383-395, 2002.

70. Katoh Y and Katoh M: WNT antagonist, SFRP1, is Hedgehog signaling target. Int J Mol Med 17: 171-175, 2006.

71. He J, Sheng T, Stelter AA, et al: Suppressing Wnt signaling by the Hedgehog pathway through SFRP1. J Biol Chem 281: 35598-35602, 2006.

72. Yauch RL, Gould SE, Scales SJ, et al: A paracrine requirement of Hedgehog signaling in cancer. Nature 455: 406-410, 2008.

73. Katoh M: Demand for user-friendly 'omics' web service utilizing next-generation supercomputer in the sequencing era. Mol Cancer Genet 1: 1-2, 2008 